\title{
Inclure l'émotionnel dans la prise en charge des douleurs
}

\author{
Jean Martin \\ Dr méd., membre de la rédaction
}

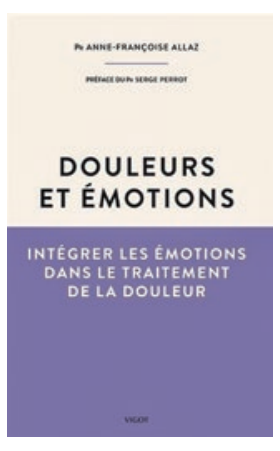

Anne-Françoise Allaz Douleurs et émotions Intégrer les émotions dans le traitement de la douleur

Paris: Editions Vigot; 2021, $127 \mathrm{p}$
La professeure Anne-Françoise Allaz a travaillé pendant 30 ans au Centre de la douleur des Hôpitaux universitaires de Genève. Elle publie un ouvrage substantiel et accessible sur un domaine qui a beaucoup gagné en importance au cours des décennies récentes.

La première partie de ce livre divisé en quatre chapitres rappelle avec limpidité les connaissances actuelles sur la douleur, ses causes (si elles sont connues), ses caractères, y compris sur le plan physio-pathologique. Suit une immersion progressive, somatique/ technique et psychologique/empathique, dans l'expérience professionnelle et académique de l'auteure, assortie de brèves vignettes cliniques. Cet exposé est marqué par l'humanité toujours nécessaire, particulièrement face à des tableaux chroniques complexes, ainsi que par le réalisme.

Est soulignée l'importance d'une prise en charge multimodale intégrée, tant dans l'évaluation de la situation que dans le développement de la démarche thérapeutique - bien loin des notions one size fits all (traitement standard) ou de la magic bullet (balle-miracle). Au-delà des aspects physiques, cette spécialiste de la douleur chronique évoque l'importance des composantes psychologiques et émotionnelles et de la relation avec le soignant. Les possibilités de traitement sont à choisir en fonction de ces composantes.

La nécessité de penser multimodal s'étend ainsi à la diversité des méthodes qui peuvent être invoquées. Cela inclut les moyens complémentaires ou alternatifs auxquels est consacrée une section. Cet intérêt basé sur les bénéfices confirmés que ces méthodes, utilisées à bon escient, peuvent apporter se manifeste aujourd'hui plus largement, en milieu universitaire aussi. Pour les moins jeunes d'entre nous, cette volonté d'ouverture et de respect à l'endroit de moyens "différents" contraste avec un certain dogmatisme médical étroit il y a un demi-siècle.

Le dernier chapitre discute l'intégration des dimensions émotionnelles dans la pratique: du côté des thérapeutes, des proches, des personnes souffrantes. On peut citer ce passage, très parlant: «Pour que la ren- contre thérapeutique soit féconde, l'intérêt pour le système de référence de l'autre est essentiel.» Un axiome peut-on dire. Ou encore: «L'accueil du patient et de sa souffrance est au centre de toute offre de soins [...] Les expériences de vie des patients - dont leurs relations avec le système de santé - ont souvent renforcé leurs craintes de ne pas être crus, ou leur impression d'être rejetés.» Certains se montrent réticents quand l'éventualité de prescription d'antidépresseurs est évoquée, à cause de la connotation "psy", alors même que ces substances sont souvent utiles dans le traitement des douleurs (pp. 76-77).

A la fin de l'ouvrage, l'auteure transmet un message capital: «Il est important de savoir expliquer que, en médecine algologique, le modèle de soin est celui de la réhabilitation, où la séquence linéaire traditionnelle diagnostic-traitement-guérison n'est pas applicable.» A propos des thérapies dites humanistes, elle cite leur initiateur Carl Rogers: «Le curieux paradoxe, c'est que quand je m'accepte comme je suis, alors je peux changer.»

Un ouvrage didactique à recommander pour toutes disciplines, tant les notions d'ouverture et de multimodalité sont fondamentales.

Le médecin de santé publique qui signe cette recension souligne l'importance de «Si des différences culturelles de perception et de réponse à la douleur sont observées, elles sont en réalité souvent battues en brèche par les déterminants sociaux. Les facteurs ethniques et culturels ont, en revanche, été associés à une prise en charge inégalitaire, sans doute en lien avec divers stéréotypes.» De quoi nous rappeler que ces inégalités sont le problème majeur de nos systèmes de santé!

Didactique, Anne-Françoise Allaz s'attelle à expliquer de manière conviviale - comme elle l'a été avec sa patientèle. On recommande la lecture de cet ouvrage structuré et bien écrit aux thérapeutes de toutes disciplines, tant les notions d'ouverture (d'esprit et pratique), dénuées de jugement, et de multimodalité sont fondamentales. Dans ce sens, Douleurs et émotions peut beaucoup apporter à un stade précoce dans la formation. 